\title{
On Caputo modification of the Hadamard fractional derivatives
}

\author{
Yusuf Y Gambo ${ }^{1,2}$, Fahd Jarad ${ }^{3 *}$, Dumitru Baleanu ${ }^{2,4,5}$ and Thabet Abdeljawad ${ }^{2,6}$
}

\footnotetext{
Correspondence: fjarad@thk.edu.tr

${ }^{3}$ Department of Logistics

Management, Faculty of

Management, University of Turkish

Aeronautical Association, Etimesgut,

Ankara, 06790, Turkey

Full list of author information is

available at the end of the article
}

\begin{abstract}
This paper is devoted to the study of Caputo modification of the Hadamard fractional derivatives. From here and after, by Caputo-Hadamard derivative, we refer to this modified fractional derivative (Jarad et al. in Adv. Differ. Equ. 2012:142, 2012, p.7). We present the generalization of the fundamental theorem of fractional calculus (FTFC) in the Caputo-Hadamard setting. Also, several new related results are presented.
\end{abstract}

Keywords: Caputo-Hadamard fractional derivatives; fundamental theorem of fractional calculus

\section{Introduction}

Fractional calculus started to be considered deeply as a powerful tool to reveal the hidden aspects of the dynamics of the complex or hypercomplex systems [1-7].

Finding new generalization of the existing fractional derivatives was always the main direction of research within this field. These generalized operators will give us new opportunities to improve the existing results from theoretical and applied viewpoints. Although the works in [8-10] played important roles in the development of the fractional calculus within the frame of the Hadamard derivative, vast and vital work in this field is still undone.

The presence of the $\delta$-differential operator $\left(\delta=x \frac{d}{d x}\right)$ in the definition of Hadamard fractional derivatives could make their study uninteresting and less applicable than RiemannLiouville and Caputo fractional derivatives. More so, this operator appears outside the integral in the definition of the Hadamard derivatives just like the usual derivative $D=\frac{d}{d x}$ is located outside the integral in the case of Riemann-Liouville, which makes the fractional derivative of a constant of these two types not equal to zero in general. The authors in [11] studied and modified the Hadamard derivatives into a more useful type using Caputo definitions.

Hadamard proposed a fractional power of the form $\left(x \frac{d}{d x}\right)^{\alpha}$. This fractional derivative is invariant with respect to dilation on the whole axis. The Hadamard approach to fractional integral was based on the generalisation of the $n$th integral [11]

$$
\left(\mathcal{J}_{a+}^{n} f\right)(x)=\int_{a}^{x} \frac{d t_{1}}{t_{1}} \int_{a}^{t_{1}} \frac{d t_{2}}{t_{2}} \cdots \int_{a}^{t_{n-1}} f\left(t_{n}\right) \frac{d t_{n}}{t_{n}} .
$$

Just like Riemann-Liouville, Hadamard derivative has its own disadvantages as well, one of which is the fact that the derivative of a constant is not equal to 0 in general. The authors in

(อ2014 Gambo et al.: licensee Springer. This is an Open Access article distributed under the terms of the Creative Commons Attribution License (http://creativecommons.org/licenses/by/2.0), which permits unrestricted use, distribution, and reproduction in any medium, provided the original work is properly cited. 
[11] resolved these problems by modifying the derivative into a more suitable one having physically interpretable initial conditions similar to the ones in the Caputo settings.

In [12-14], the authors recovered the concepts of fractional integrals and fractional derivatives in different forms and introduced a new version of FTFC in Caputo settings, which is regarded as a generalization of the classical fundamental theorem of calculus. This ignites our curiosity in the possibility of generalizing FTFC in the sense our new definitions given in [11] as Hadamard and Riemann-Liouville (for example) cannot be used for this generalization (see Section 3). Using the generalization or otherwise, we then formulate new results and theorems.

We study much of this modified derivative thereby formulating some important theorems and results. The Caputo-Hadamard fractional derivatives are used to develop the FTFC, and then the new results are applied in the formulation of some other theorems. As we shall see later, some interesting properties of the modified derivatives are necessary in order to formulate some important outcomes. Section 2 gives some definitions and known results which have been used in this paper, whilst both Sections 3 and 4 are devoted to the original results. Section 5 concludes the paper.

\section{Auxiliary results}

Below, we begin with some basic definitions and results.

Let $0 \leq a \leq b \leq \infty$ be finite or infinite interval of the half-axis $\mathbb{R}^{+}$. The Hadamard fractional integrals of order $\alpha \in \mathbb{C}$ are defined [5] by

$$
\begin{array}{ll}
\left(\mathcal{J}_{a+}^{\alpha} \varphi\right)(x)=\frac{1}{\Gamma(\alpha)} \int_{a}^{x}\left(\log \frac{x}{t}\right)^{\alpha-1} \varphi(t) \frac{d t}{t}, & a<x<b, \\
\left(\mathcal{J}_{b-}^{\alpha} \varphi\right)(x)=\frac{1}{\Gamma(\alpha)} \int_{x}^{b}\left(\log \frac{t}{x}\right)^{\alpha-1} \varphi(t) \frac{d t}{t}, & a<x<b .
\end{array}
$$

The left-sided and right-sided Hadamard fractional derivatives of order $\alpha \in \mathbb{C}$ with $\mathfrak{R}(\alpha) \geq$ 0 on $(a, b)$ and $a<x<b$ are defined by

$$
\begin{aligned}
& \left(\mathfrak{D}_{a+}^{\alpha} \varphi\right)(x)=\delta^{n}\left(\mathcal{J}_{a+}^{n-\alpha} \varphi\right)(x)=\left(x \frac{d}{d x}\right)^{n} \frac{1}{\Gamma(n-\alpha)} \int_{a}^{x}\left(\log \frac{x}{t}\right)^{n-\alpha-1} \frac{\varphi(t) d t}{t}, \\
& \left(\mathfrak{D}_{b-}^{\alpha} \varphi\right)(x)=(-\delta)^{n}\left(\mathcal{J}_{b-}^{n-\alpha} \varphi\right)(x)=\left(-x \frac{d}{d x}\right)^{n} \frac{1}{\Gamma(n-\alpha)} \int_{x}^{b}\left(\log \frac{t}{x}\right)^{n-\alpha-1} \frac{\varphi(t) d t}{t},
\end{aligned}
$$

where $n=[\Re(\alpha)]+1$.

Property 1 [5, p.112] If $\mathfrak{R}(\alpha)>0, \mathfrak{R}(\beta)>0$ and $0<a<b<\infty$, then we have

$$
\begin{aligned}
& \left(\mathcal{J}_{a+}^{\alpha}\left(\log \frac{t}{a}\right)^{\beta-1}\right)(x)=\frac{\Gamma(\beta)}{\Gamma(\beta+\alpha)}\left(\log \frac{x}{a}\right)^{\beta+\alpha-1}, \\
& \left(\mathfrak{D}_{a+}^{\alpha}\left(\log \frac{t}{a}\right)^{\beta-1}\right)(x)=\frac{\Gamma(\beta)}{\Gamma(\beta-\alpha)}\left(\log \frac{x}{a}\right)^{\beta-\alpha-1}, \\
& \left(\mathcal{J}_{b-}^{\alpha}\left(\log \frac{b}{t}\right)^{\beta-1}\right)(x)=\frac{\Gamma(\beta)}{\Gamma(\beta+\alpha)}\left(\log \frac{b}{x}\right)^{\beta+\alpha-1}, \\
& \left(\mathfrak{D}_{b-}^{\alpha}\left(\log \frac{b}{t}\right)^{\beta-1}\right)(x)=\frac{\Gamma(\beta)}{\Gamma(\beta-\alpha)}\left(\log \frac{b}{x}\right)^{\beta-\alpha-1} .
\end{aligned}
$$


Lemma 1 [5, pp.114-116] Let $\alpha, \beta \in \mathbb{C}$ such that $\mathfrak{R}(\alpha)>\mathfrak{R}(\beta)>0$.

(a) If $0<a<b<\infty$ and $1 \leq p<\infty$, then for $\varphi \in L^{p}(a, b)$,

$$
\mathfrak{D}_{a^{+}}^{\beta} \mathcal{J}_{a_{+}}^{\alpha} \varphi=\mathcal{J}_{a+}^{\alpha-\beta} \varphi \quad \text { and } \quad \mathfrak{D}_{b_{-}}^{\beta} \mathcal{J}_{b-}^{\alpha} \varphi=\mathcal{J}_{b-}^{\alpha-\beta} \varphi ;
$$

(b)

$$
\mathcal{J}_{a^{+}}^{\alpha} \mathcal{J}_{a^{+}}^{\beta} \varphi=\mathcal{J}_{a_{+}}^{\alpha+\beta} \varphi \quad \text { and } \quad \mathcal{J}_{b_{-}}^{\alpha} \mathcal{J}_{b-}^{\beta} \varphi=\mathcal{J}_{b-}^{\alpha+\beta} \varphi
$$

Equations (10) and (11) are called semigroup properties of Hadamard fractional integrals and derivatives.

The Caputo-type modifications of the left-sided and right-sided Hadamard fractional derivatives are defined [11] respectively by

$$
\begin{aligned}
& { }^{C} \mathfrak{D}_{a+}^{\alpha} \varphi(x)=\mathfrak{D}_{a+}^{\alpha}\left[\varphi(t)-\sum_{k=0}^{n-1} \frac{\delta^{k} \varphi(a)}{k !}\left(\log \frac{t}{a}\right)^{k}\right](x), \\
& C_{\mathfrak{D}_{b-}^{\alpha}} \varphi(x)=\mathfrak{D}_{b-}^{\alpha}\left[\varphi(t)-\sum_{k=0}^{n-1} \frac{(-1)^{k} \delta^{k} \varphi(b)}{k !}\left(\log \frac{b}{t}\right)^{k}\right](x) .
\end{aligned}
$$

Here $\mathfrak{R}(\alpha) \geq 0, n=[\Re(\alpha)+1], 0<a<b<\infty$ and

$$
\varphi(x) \in A C_{\delta}^{n}[a, b]=\left\{\varphi:[a, b] \rightarrow \mathbb{C}: \delta^{(n-1)} \varphi(x) \in A C[a, b], \delta=x \frac{d}{d x}\right\} .
$$

In particular, if $0<\mathfrak{R}(\alpha)<1$, then

$$
\begin{aligned}
C_{\mathfrak{D}_{a+}^{\alpha}}^{\alpha} \varphi(x) & =\mathfrak{D}_{a+}^{\alpha}[\varphi(t)-\varphi(a)](x), \\
{ }^{C} \mathfrak{D}_{b_{-}}^{\alpha} \varphi(x) & =\mathfrak{D}_{b-}^{\alpha}[\varphi(t)-\varphi(b)](x) .
\end{aligned}
$$

Theorem 1 [11, p.4] Let $\Re(\alpha) \geq 0, n=[\Re(\alpha)]+1$ and $\varphi \in A C_{\delta}^{n}[a, b], 0<a<b<\infty$. Then ${ }^{C} \mathfrak{D}_{a_{+}}^{\alpha} \varphi(x)$ and ${ }^{C} \mathfrak{D}_{b-}^{\alpha} \varphi(x)$ exist everywhere on $[a, b]$ and

(a) if $\alpha \notin \mathbb{N}_{0}$,

$$
\begin{aligned}
& C_{\mathfrak{D}_{a+}^{\alpha}}^{\alpha} \varphi(x)=\frac{1}{\Gamma(n-\alpha)} \int_{a}^{x}\left(\log \frac{x}{t}\right)^{n-\alpha-1} \delta^{n} \varphi(t) \frac{d t}{t}=\mathcal{J}_{a+}^{n-\alpha} \delta^{n} \varphi(x), \\
& { }^{C} \mathfrak{D}_{b-}^{\alpha} \varphi(x)=\frac{(-1)^{n}}{\Gamma(n-\alpha)} \int_{x}^{b}\left(\log \frac{t}{x}\right)^{n-\alpha-1} \delta^{n} \varphi(t) \frac{d t}{t}=(-1)^{n} \mathcal{J}_{b-}^{n-\alpha} \delta^{n} \varphi(x) ;
\end{aligned}
$$

(b) if $\alpha=n \in \mathbb{N}_{0}$,

$$
{ }^{C} \mathfrak{D}_{a+}^{\alpha} \varphi(x)=\delta^{n} \varphi(x), \quad{ }^{C} \mathfrak{D}_{b-}^{\alpha} \varphi(x)=(-1)^{n} \delta^{n} \varphi(x) .
$$

In particular,

$$
{ }^{C} \mathfrak{D}_{a+}^{0} \varphi(x)={ }^{C} \mathfrak{D}_{b-}^{0} \varphi(x)=\varphi(x) .
$$


Lemma $2[11$, p.5] Let $\mathfrak{R}(\alpha)>0, n=[\Re(\alpha)]+1$ and $\varphi \in C[a, b]$.

If $\mathfrak{R}(\alpha) \neq 0$ or $\alpha \in \mathbb{N}$, then

$$
C^{C} \mathfrak{D}_{a^{+}}^{\alpha}\left(\mathcal{J}_{a+}^{\alpha} \varphi\right)(x)=\varphi(x), \quad{ }^{C} \mathfrak{D}_{b-}^{\alpha}\left(\mathcal{J}_{b-}^{\alpha} \varphi\right)(x)=\varphi(x) .
$$

Lemma $3[11, \mathrm{p} .6]$ Let $\varphi \in A C_{\delta}^{n}[a, b]$ or $C_{\delta}^{n}[a, b]$ and $\alpha \in \mathbb{C}$, then

$$
\begin{aligned}
& \mathcal{J}_{a+}^{\alpha}\left({ }^{C} \mathfrak{D}_{a+}^{\alpha} \varphi\right)(x)=\varphi(x)-\sum_{k=0}^{n-1} \frac{\delta^{k} \varphi(a)}{k !}\left(\log \frac{x}{a}\right)^{k}, \\
& \mathcal{J}_{b-}^{\alpha}\left({ }^{C} \mathfrak{D}_{b-}^{\alpha} \varphi\right)(x)=\varphi(x)-\sum_{k=0}^{n-1} \frac{\delta^{k} \varphi(b)}{k !}\left(\log \frac{b}{x}\right)^{k} .
\end{aligned}
$$

\section{FTFC in the Caputo-Hadamard setting}

The fundamental theorem of calculus FTC

$$
\frac{d}{d x} \int_{a}^{x} f(t) d t=f(x) \text { and } \int_{a}^{b} d t \frac{d}{d t} F(t)=F(b)-F(a)=\left.F(t)\right|_{a} ^{b}
$$

replaces tedious computations of the limit of sums of rectangular areas with a more easier way of finding an anti-derivative.

In the fractional case, Riemann-Liouville as well as Hadamard integro-differentiation (for example) do not have generalization of the fundamental theorem of fractional calculus (FTFC) in the form of (24), i.e.,

$$
\begin{array}{ll}
\left({ }_{a} I_{b}^{\alpha} \mathfrak{D}_{a+}^{\alpha} \varphi\right)(x) \neq \varphi(b)-\varphi(a), & \left({ }_{a} I_{b}^{\alpha} \mathfrak{D}_{b-}^{\alpha} \varphi\right)(x) \neq \varphi(a)-\varphi(b), \\
\left({ }_{a} \mathcal{J}_{b}^{\alpha} \mathfrak{D}_{a+}^{\alpha} \varphi\right)(x) \neq \varphi(b)-\varphi(a), & \left({ }_{a} \mathcal{J}_{b}^{\alpha} \mathfrak{D}_{b-}^{\alpha} \varphi\right)(x) \neq \varphi(a)-\varphi(b),
\end{array}
$$

where

$$
\left({ }_{a} I_{x}^{\alpha} \varphi\right)(x)=\frac{1}{\Gamma(\alpha)} \int_{a}^{x} \frac{\varphi(t) d t}{(x-t)^{1-\alpha}}, \quad x>a, \mathfrak{R}(\alpha)>0
$$

and

$$
\left(\mathfrak{D}_{a+}^{\alpha} \varphi\right)(x)=\left(\frac{d}{d x}\right)^{n}\left(I_{a+}^{n-\alpha} \varphi\right)(x), \quad x>a, n=[\Re(\alpha)+1]
$$

are the left-sided Riemann-Liouville fractional integral and the fractional derivative, respectively. While the Hadamard fractional integral and the fractional derivative, ${ }_{a} \mathcal{J}_{x}^{\alpha}$ and $\mathfrak{D}_{a+}^{\alpha}$ respectively, are given by (2) to (5).

The reason to the above assertion is the fact that the differential operators $D^{n}=\left(\frac{d}{d x}\right)^{n}$ and $\delta^{n}=\left(x \frac{d}{d x}\right)^{n}$ used in the definitions of Riemann-Liouville and Hadamard fractional derivatives, respectively, appear outside the integrals; and therefore, as those operators and the integrals are not commutative, the semigroup properties for integrals (10) and Lemma 2.3 of [5] cannot be applied, i.e.,

$$
\begin{aligned}
& \left(I_{a+}^{\alpha} \mathfrak{D}_{a+}^{\alpha} \varphi\right)(x)=I_{a+}^{\alpha} D^{n} I_{a+}^{n-\alpha} \varphi(x) \neq I_{a+}^{\alpha} I_{a+}^{n-\alpha} D^{n} \varphi(x), \\
& \left(\mathcal{J}_{a+}^{\alpha} \mathfrak{D}_{a+}^{\alpha} \varphi\right)(x)=\mathcal{J}_{a+}^{\alpha} \delta^{n} \mathcal{J}_{a+}^{n-\alpha} \varphi(x) \neq \mathcal{J}_{a+}^{\alpha} \mathcal{J}_{a+}^{n-\alpha} \delta^{n} \varphi(x) .
\end{aligned}
$$


However, from Theorem 2.3 of [5], we have

$$
\left(\mathcal{J}_{a+}^{\alpha} \mathfrak{D}_{a+}^{\alpha} \varphi\right)(x)=\varphi(x)-\sum_{j=1}^{n} \frac{\left(\delta^{n-j}\left(\mathcal{J}_{a+}^{n-\alpha} \varphi\right)\right)(a)}{\Gamma(\alpha-j+1)}\left(\log \frac{x}{a}\right)^{\alpha-j}
$$

In particular, if $0<\mathfrak{R}(\alpha)<1$, then $n=1$ and

$$
\left(\mathcal{J}_{a+}^{\alpha} \mathfrak{D}_{a+}^{\alpha} \varphi\right)(x)=\varphi(x)-\frac{\left(\mathcal{J}_{a+}^{1-\alpha} \varphi\right)(a)}{\Gamma(\alpha)}\left(\log \frac{x}{a}\right)^{\alpha-1}
$$

If $\alpha=1$, then

$$
\left(\mathcal{J}_{a+}^{1} \mathfrak{D}_{a+}^{1} \varphi\right)(x)=\varphi(x)-\varphi(a),
$$

which implies

$$
\left({ }_{a} \mathcal{J}_{b}^{1} \mathfrak{D}_{a+}^{1} \varphi\right)(x)=\varphi(b)-\varphi(a)
$$

Thus, (30) cannot be considered as the fractional generalization of FTC in the form of (24). Similarly, using Lemma 2.5 of [5], we can see that Riemann-Liouville fractional integrals and derivatives cannot be used to generalize FTFC in the form of (24) as well.

On the other hand, replacing the Riemann-Liouville fractional derivative with the Caputo fractional derivative, it was shown (in, for example, [12, 13] and [14]) that

$$
\left({ }_{a} I_{b}^{\alpha C} \mathfrak{D}_{a+}^{\alpha} \varphi\right)(x)=\varphi(b)-\varphi(a) .
$$

In most cases, we would only be using the left-sided definitions of fractional derivatives or integrals where the definitions are quite similar to the right-sided ones. Therefore (33) can be considered as a fractional generalization of FTC in the form of (24).

In the next theorem, we give the FTFC in the Caputo-Hadamard setting.

Theorem 2 (Fundamental theorem of fractional calculus) Let $\alpha \in \mathbb{C}$ with $\mathfrak{R}(\alpha) \geq 0$ and $n=[\Re(\alpha)]+1$. Let $\varphi(x) \in A C_{\delta}^{n}[a, b], 0<a<b<\infty$.

(a) If $\Phi(x)=\mathcal{J}_{a+}^{\alpha} \varphi(x)$ or $\Phi(x)=\mathcal{J}_{b-}^{\alpha} \varphi(x) \forall x \in[a, b]$, then

$$
{ }^{C} \mathfrak{D}_{a+}^{\alpha} \Phi(x)=\varphi(x), \quad{ }^{C} \mathfrak{D}_{b-}^{\alpha} \Phi(x)=\varphi(x) .
$$

(b)

$$
{ }_{a} \mathcal{J}_{b}^{\alpha C} \mathfrak{D}_{a+}^{\alpha} \Phi(x)=\Phi(b)-\Phi(a), \quad{ }_{a} \mathcal{J}_{b}^{\alpha C} \mathfrak{D}_{b-}^{\alpha} \Phi(x)=\Phi(a)-\Phi(b) .
$$

Proof (a) Using Lemma 2.4 of [11], it can be seen that the Hadamard fractional integrals and the Caputo-Hadamard fractional derivatives are inverse operations,

$$
\left({ }^{C} \mathfrak{D}_{a+}^{\alpha} \mathcal{J}_{a+}^{\alpha}\right) \varphi(x)=\varphi(x), \quad\left({ }^{C} \mathfrak{D}_{b-}^{\alpha} \mathcal{J}_{b-}^{\alpha}\right) \varphi(x)=\varphi(x)
$$

Thus, if $\Phi(x)=\mathcal{J}_{a+}^{\alpha} \varphi(x)$ or $\Phi(x)=\mathcal{J}_{b-}^{\alpha} \varphi(x)$, then we have (34). 
(b) Using (17), we have

$$
\mathcal{J}_{a+}^{\alpha C} \mathfrak{D}_{a+}^{\alpha} \Phi(x)=\mathcal{J}_{a+}^{\alpha} \mathcal{J}_{a+}^{n-\alpha} \delta^{n} \Phi(x)
$$

In this case we can apply the semigroup proper (10), unlike in the cases of Hadamard and Riemann-Liouville fractional derivatives where $\delta^{n}$ and $D^{n}$, respectively, are located outside the integrals.

Therefore

$$
\mathcal{J}_{a+}^{\alpha}{ }^{C} \mathfrak{D}_{a+}^{\alpha} \Phi(x)=\mathcal{J}_{a+}^{n} \delta^{n} \Phi(x)
$$

In particular, if $n=1$, then

$$
\begin{aligned}
\mathcal{J}_{a+}^{\alpha C} \mathfrak{D}_{a+}^{\alpha} \Phi(x) & =\mathcal{J}_{a+}^{1} \delta^{1} \Phi(x) \\
& =\frac{1}{\Gamma(1)} \int_{a}^{x} \frac{d t}{t} \cdot t \frac{d}{d t} \Phi(t) \\
& =\int_{a}^{x} d t \frac{d}{d t} \Phi(t)=\Phi(x)-\Phi(a),
\end{aligned}
$$

which implies that

$$
{ }_{a} \mathcal{J}_{b}^{\alpha C} \mathfrak{D}_{a+}^{\alpha} \Phi(x)=\int_{a}^{b} d t \frac{d}{d t} \Phi(t)=\Phi(b)-\Phi(a) .
$$

Alternatively, using Lemma 2.5 of [11], we have

$$
\mathcal{J}_{a+}^{\alpha C} \mathfrak{D}_{a+}^{\alpha} \Phi(x)=\Phi(x)-\sum_{j=0}^{n-1} \frac{\delta^{j} \Phi(a)}{\Gamma(j+1)}\left(\log \frac{x}{a}\right)^{j} .
$$

In particular, if $0<\mathfrak{R}(\alpha)<1$, then $n=1$ and $\Phi(x) \in A C_{\delta}[a, b]$ or $\Phi(x) \in C_{\delta}[a, b]$. Thus

$$
\mathcal{J}_{a+}^{\alpha C} \mathfrak{D}_{a+}^{\alpha} \Phi(x)=\Phi(x)-\Phi(a)
$$

Hence ${ }_{a} \mathcal{J}_{b}^{\alpha C} \mathfrak{D}_{a+}^{\alpha} \Phi(x)$ gives (35). The right-sided case can be proven in a similar way.

Lemma 4 Let $\alpha \in \mathbb{C}$ with $\mathfrak{R}(\alpha) \geq 0$ and $n=[\Re(\alpha)]+1$. If $\varphi(x) \in A C_{\delta}^{n}[a, b]$, where $0<a<$ $b<\infty$, then

$$
\begin{aligned}
& \varphi(x)=\varphi(a)+\frac{{ }^{C} \mathfrak{D}_{a+}^{\alpha} \varphi(\xi)}{\Gamma(\alpha+1)}\left(\log \frac{x}{a}\right)^{\alpha}, \quad \xi \in(a, x) \quad \text { or } \\
& \varphi(x)=\varphi(b)+\frac{{ }^{C} \mathfrak{D}_{b-}^{\alpha} \varphi(\xi)}{\Gamma(\alpha+1)}\left(\log \frac{b}{x}\right)^{\alpha}, \quad \xi \in(x, b) .
\end{aligned}
$$

This is where we make the first use of Theorem 2.

Proof Using (2) and (40), we obtain

$$
\frac{1}{\Gamma(\alpha)} \int_{a}^{x}\left(\log \frac{x}{t}\right)^{\alpha-1} C_{\mathfrak{D}_{a+}^{\alpha}} \varphi(t) \frac{d t}{t}=\varphi(x)-\varphi(a),
$$


where the function is ${ }^{C} \mathfrak{D}_{a^{+}}^{\alpha} \varphi(t)$. Applying the mean value theorem for integrals [15, p.287], we have

$$
{ }^{C} \mathfrak{D}_{a+}^{\alpha} \varphi(\xi) \frac{1}{\Gamma(\alpha)} \int_{a}^{x}\left(\log \frac{x}{t}\right)^{\alpha-1} \frac{d t}{t}=\varphi(x)-\varphi(a), \quad \xi \in(a, x) .
$$

The left-hand side of (42) contains the Hadamard fractional integral of the function $\varphi(t)=$ 1, i.e., $\mathcal{J}_{a+}^{\alpha}(1)$. Using (6) implies $\beta=1$ and thus (42) becomes

$$
\frac{{ }^{C} \mathfrak{D}_{a+}^{\alpha} \varphi(\xi)}{\Gamma(\alpha+1)}\left(\log \frac{x}{t}\right)^{\alpha}=\varphi(x)-\varphi(a), \quad \xi \in(a, x) .
$$

Rearranging (43) gives (41). This completes the proof.

Note that the right-sided case can also be proven in a similar way.

Lemma 5 If $\alpha \in \mathbb{C}$ with $\mathfrak{R}(\alpha) \geq 0, n=[\Re(\alpha)]+1$ and $k, m \in \mathbb{N}, \varphi(x) \in A C_{\delta}^{n}[a, b], 0<a<$ $b<\infty$, then

$$
\begin{aligned}
& \left(\mathcal{J}_{a+}^{\alpha}\right)^{k}\left({ }^{C} \mathfrak{D}_{a+}^{\alpha}\right)^{m} \varphi(x)=\frac{\left({ }^{C} \mathfrak{D}_{a+}^{\alpha}\right)^{m} \varphi(\xi)}{\Gamma(k \alpha+1)}\left(\log \frac{x}{a}\right)^{k \alpha}, \quad \xi \in(a, x) \quad \text { or } \\
& \left(\mathcal{J}_{b-}^{\alpha}\right)^{k}\left({ }^{C} \mathfrak{D}_{b-}^{\alpha}\right)^{m} \varphi(x)=\frac{\left({ }^{C} \mathfrak{D}_{b-}^{\alpha}\right)^{m} \varphi(\xi)}{\Gamma(k \alpha+1)}\left(\log \frac{b}{x}\right)^{k \alpha}, \quad \xi \in(x, b) .
\end{aligned}
$$

Proof The proof is similar to the proof of Lemma 4. Observe that the sequential integral

$$
\left(\mathcal{J}_{a+}^{\alpha}\right)^{k}=\underbrace{\mathcal{J}_{a+}^{\alpha} \mathcal{J}_{a+}^{\alpha} \cdots \mathcal{J}_{a+}^{\alpha}}_{k \text {-times }}
$$

can be written as the $\mathcal{J}_{a+}^{k \alpha}$ with order $k \alpha$ by the semigroup property (10). Thus,

$$
\left(\mathcal{J}_{a+}^{\alpha}\right)^{k}\left({ }^{C} \mathfrak{D}_{a+}^{\alpha}\right)^{m} \varphi(x)=\frac{1}{\Gamma(k \alpha)} \int_{a}^{x}\left(\log \frac{x}{t}\right)^{k \alpha-1}\left({ }^{C} \mathfrak{D}_{a+}^{\alpha}\right)^{m} \varphi(t) \frac{d t}{t} .
$$

Applying the mean value theorem for integral and simplifying as before, we obtain (44).

Lemma $6 \mathfrak{R}(\alpha) \geq 0$ and $n=[\Re(\alpha)]+1$, if $\varphi$ is a function such that ${ }^{C} \mathfrak{D}_{a_{+}}^{\alpha} \varphi$ and $\mathfrak{D}_{a+}^{\alpha} \varphi$ exist, then

$$
C^{C} \mathfrak{D}_{a+}^{\alpha} \varphi(x)=\mathfrak{D}_{a+}^{\alpha} \varphi(x)-\sum_{k=0}^{n-1} \frac{\delta^{k} \varphi(a)}{\Gamma(k-\alpha+1)}\left(\log \frac{x}{a}\right)^{k-\alpha},
$$

and when $0<\mathfrak{R}(\alpha)<1$, then

$$
{ }^{C} \mathfrak{D}_{a+}^{\alpha} \varphi(x)=\mathfrak{D}_{a+}^{\alpha} \varphi(x)-\frac{\varphi(a)}{\Gamma(1-\alpha)}\left(\log \frac{x}{a}\right)^{-\alpha} .
$$


Proof Using (7) and (12), we obtain

$$
\begin{aligned}
C_{\mathfrak{D}_{a+}^{\alpha} \varphi(x)} & =\mathfrak{D}_{a+}^{\alpha} \varphi(x)-\sum_{k=0}^{n-1} \frac{\delta^{k} \varphi(a)}{k !}\left(\mathfrak{D}_{a+}^{\alpha}\left(\log \frac{t}{a}\right)^{k}\right)(x) \\
& =\mathfrak{D}_{a+}^{\alpha} \varphi(x)-\sum_{k=0}^{n-1} \frac{\delta^{k} \varphi(a)}{\Gamma(k+1)} \cdot \frac{\Gamma(k+1)}{\Gamma(k-\alpha+1)}\left(\log \frac{x}{a}\right)^{k-\alpha} .
\end{aligned}
$$

Thus we have (47). Then if $0<\mathfrak{R}(\alpha)<1$, implies $n=1$ and from (47), we have (48). We can get an immediate consequence of Lemma 6 .

Corollary 1 Under the conditions of Lemma 6,

$$
{ }^{C} \mathfrak{D}_{a+}^{\alpha} \varphi(x)=\mathfrak{D}_{a+}^{\alpha} \varphi(x)
$$

if and only if $\varphi$ has an $n$-fold zero at a, i.e., if and only if

$$
\delta^{j} \varphi(a)=0, \quad j=0,1, \ldots, n-1 .
$$

The proof is straightforward.

Now, it is known [11, Theorem 2.2] that if $\Re(\alpha) \geq 0$ and $\alpha \notin \mathbb{N}_{0}=\{0,1,2 \ldots\}$ such that $n=[\Re(\alpha)]+1$, then

$$
{ }^{C} \mathfrak{D}_{a+}^{\alpha} \varphi(a)=0 \quad \text { and } \quad{ }^{C} \mathfrak{D}_{b-}^{\alpha} \varphi(b)=0 .
$$

This fact disallows us to obtain (for example) a fractional Taylor series using the fractional derivatives evaluated at these points. Otherwise, we can have a series expansion in the form

$$
\varphi(x)=\sum_{j=0}^{m} \frac{\left({ }^{C} \mathfrak{D}_{a+}^{\alpha}\right)^{j} \varphi(a)}{\Gamma(j \alpha+1)}\left(\log \frac{x}{a}\right)^{j \alpha}+\mathcal{R}_{m}(a, x),
$$

where

$$
\mathcal{R}_{m}(x, a)=\frac{\left({ }^{C} \mathfrak{D}_{a+}^{\alpha}\right)^{(m+1)} \varphi(\xi)}{\Gamma((m+1) \alpha+1)}\left(\log \frac{x}{a}\right)^{(m+1) \alpha}, \quad \xi \in[a, x]
$$

is the remainder of the terms in the expansion.

However, we may relax the conditions on $\varphi$ in Corollary 1 as in the next result.

Lemma 7 Let $\mathfrak{R}(\alpha) \geq 0$ and $n=[\Re(\alpha)]+1$ such that $\alpha \notin \mathbb{N}_{0}$ and $\varphi(x) \in A C_{\delta}^{n}[a, b]$. More so, suppose that ${ }^{C} \mathfrak{D}_{a_{+}}^{\beta} \varphi(x)$ is continuous on $[a, b]$ for some $\beta \in(\alpha, n)$. Then ${ }^{C} \mathfrak{D}_{a+}^{\alpha} \varphi(x)$ is continuous and ${ }^{C} \mathfrak{D}_{a^{+}}^{\alpha} \varphi(a)=0$.

Proof Using (17), we obtain

$$
{ }^{C} \mathfrak{D}_{a+}^{\alpha} \varphi(x)=\mathcal{J}_{a+}^{n-\alpha} \delta^{n} \varphi(x)=\mathcal{J}_{a^{+}}^{\beta-\alpha} \mathcal{J}_{a+}^{n-\beta} \delta^{n} \varphi(x)=\mathcal{J}_{a^{+}}^{\beta-\alpha} C_{\mathfrak{D}_{a+}^{\beta}}^{\beta} \varphi(x) .
$$

Thus, ${ }^{C} \mathfrak{D}_{a^{+}}^{\alpha} \varphi(x)$ is continuous and ${ }^{C} \mathfrak{D}_{a+}^{\alpha} \varphi(a)=\mathcal{J}_{a^{+}}^{\beta-\alpha}{ }^{C} \mathfrak{D}_{a_{+}}^{\beta} \varphi(a)=0$ by (51). This completes the proof of the lemma. 


\section{Semigroup properties of Caputo-Hadamard operators}

We present the first proof of the semigroup properties of Caputo-Hadamard fractional derivatives. The Caputo version of this result is seen in [16] and [17].

Theorem 3 (Semigroup property for Caputo-Hadamard derivatives) Let $\varphi(x) \in C_{\delta}^{m+n}[a$, $b], 0<a<b<\infty$. Moreover, let $\alpha \geq 0, \beta \geq 0$ such that $n-1<\alpha \leq n, m-1<\beta \leq m$. Then

$$
{ }^{C} \mathfrak{D}_{a+}^{\alpha}{ }^{C} \mathfrak{D}_{a+}^{\beta} \varphi(x)={ }^{C} \mathfrak{D}_{a+}^{\alpha+\beta} \varphi(x) .
$$

Proof Without loss of generality, let $m \geq n$. Thus, $m=n+k, k \in\{0,1,2, \ldots\}$. Since $\alpha+$ $\beta \leq m+n$, then by definitions and using semigroup properties for Hadamard fractional integrals (10), we have

$$
\begin{aligned}
& { }^{C} \mathfrak{D}_{a+}^{\alpha}{ }^{C} \mathfrak{D}_{a_{+}}^{\beta} \varphi(x)=\mathcal{J}_{a+}^{n-\alpha} \delta^{n C} \mathfrak{D}_{a_{+}}^{\beta} \varphi(x)=\mathcal{J}_{a+}^{n-\alpha} \delta^{n} \mathcal{J}_{a+}^{m-\beta} \delta^{m} \varphi(x) \\
& =\mathcal{J}_{a+}^{n-\alpha} \delta^{n} \mathcal{J}_{a+}^{n+k-\beta} \delta^{n+k} \varphi(x)=\mathcal{J}_{a+}^{n-\alpha} \delta^{n} \mathcal{J}_{a+}^{n-\beta} \mathcal{J}_{a+}^{k}{ }^{n+k} \varphi(x) \\
& =\mathcal{J}_{a+}^{n-\alpha} \mathfrak{D}_{a+}^{\beta} \mathcal{J}_{a+}^{k} \delta^{n+k} \varphi(x)=\mathcal{J}_{a^{+}}^{n-\alpha-\beta} \mathcal{J}_{a+}^{\beta} \mathfrak{D}_{a+}^{\beta} \mathcal{J}_{a^{+}}^{k} \delta^{n+k} \varphi(x) \text {. }
\end{aligned}
$$

Then, using (29) with $y(x)$ taken as $\mathcal{J}_{a+}^{k} \delta^{n+k} \varphi(x)$, we obtain

$$
\begin{aligned}
{ }^{C} \mathfrak{D}_{a_{+}}^{\alpha}{ }^{C} \mathfrak{D}_{a+}^{\beta} \varphi(x) & =\mathcal{J}_{a^{+}}^{n-\alpha-\beta}\left[\mathcal{J}_{a^{+}}^{k} \delta^{n+k} \varphi(x)-\sum_{j=1}^{n} \frac{\left(\delta^{n-j}\left(\mathcal{J}_{a+}^{n-\beta} \mathcal{J}_{a+}^{k} \delta^{n+k} \varphi\right)(a)\right)}{\Gamma(\beta-j+1)}\left(\log \frac{x}{a}\right)^{\beta-j}\right] \\
& =\mathcal{J}_{a^{+}}^{n-(\alpha+\beta)}\left[\mathcal{J}_{a^{+}}^{k} \delta^{n+k} \varphi(x)-\sum_{j=1}^{n} \frac{\left(\delta^{n-j C} \mathfrak{D}_{a+}^{\beta} \varphi(a)\right)}{\Gamma(\beta-j+1)}\left(\log \frac{x}{a}\right)^{\beta-j}\right] \\
& =\mathcal{J}_{a^{+}}^{n-(\alpha+\beta)}\left(\mathcal{J}_{a+}^{k} \delta^{n+k} \varphi(x)\right) \text { by (51) } \\
& =\mathcal{J}_{a^{+}}^{n+k-(\alpha+\beta)} \delta^{n+k} \varphi(x)={ }^{C} \mathfrak{D}_{a_{+}}^{\alpha+\beta} \varphi(x) .
\end{aligned}
$$

This ends the proof.

Example It is important to note that the purpose of this example is to show that Theorem 3 does not hold in general if the Hadamard fractional derivative is to be used instead of the Caputo-Hadamard fractional derivative. Then it suffices to present a single case where this assertion is true. Suppose that we have the function $\varphi(x)=1$ with $\alpha=\frac{2}{3}, \beta=1$ and $a=1$. Thus by definitions, the left-hand side gives

$$
\mathfrak{D}_{1+}^{2 / 3} \mathfrak{D}_{1+}^{1}(1)=\mathfrak{D}_{1+}^{2 / 3} \delta^{1}(1)=0
$$

The right-hand side would be

$$
\mathfrak{D}_{1+}^{5 / 3}(1)=\delta^{2} \mathcal{J}_{1+}^{1 / 3}(1)=\delta^{2} \frac{1}{\Gamma\left(\frac{4}{3}\right)}(\log x)^{1 / 3} \neq 0,
$$

where $\delta=x \frac{d}{d x}$. However, in the case of Caputo-Hadamard derivative, the left-hand side becomes

$$
{ }^{C} \mathfrak{D}_{1+}^{2 / 3 C} \mathfrak{D}_{1+}^{1}(1)={ }^{C} \mathfrak{D}_{1+}^{1 / 2} \delta^{1}(1)=0,
$$


and by (40) of Property 2.6 in [11], the right-hand side gives

$$
{ }^{C} \mathfrak{D}_{1+}^{5 / 3}(1)=0 .
$$

In the next lemma, we give the generalization of Theorem 3.

Lemma 8 For $\varphi(x) \in C_{\delta}^{n}[a, b], 0<a<b<\infty$,

$$
{ }^{C} \mathfrak{D}_{a^{+}}^{\alpha_{1} C} \mathfrak{D}_{a+}^{\alpha_{2}} \ldots{ }^{C} \mathfrak{D}_{a+}^{\alpha_{m}} \varphi(x)={ }^{C} \mathfrak{D}^{\sum_{a+}^{m}{ }^{m} \alpha_{j}} \varphi(x),
$$

where $\alpha_{j} \geq 0, n_{j-1}<\alpha_{j} \leq n_{j}$ and $\sum_{j=1}^{m} \alpha_{j} \leq n \forall j=\{1,2, \ldots, m\}$.

Proof The proof follows immediately from Theorem 3 and using mathematical induction.

Theorem 4 Let $\varphi(x) \in C_{\delta}^{n}[a, b], 0<a<b<\infty$ and $\alpha \in \mathbb{C}, \beta \in \mathbb{C}$ such that $\mathfrak{R}(\alpha) \geq 0$, $\mathfrak{R}(\beta) \geq 0$. Then

$$
{ }^{C} \mathfrak{D}_{a^{+}}^{\alpha} \mathcal{J}_{a+}^{\beta} \varphi(x)=\mathcal{J}_{a^{+}}^{\beta-\alpha} \varphi(x) \text { and }{ }^{C} \mathfrak{D}_{b-}^{\alpha} \mathcal{J}_{b-}^{\beta} \varphi(x)=\mathcal{J}_{b-}^{\beta-\alpha} \varphi(x) .
$$

Proof

$$
\begin{aligned}
{ }^{C} \mathfrak{D}_{a+}^{\alpha} \mathcal{J}_{a+}^{\beta} \varphi(x) & =\mathcal{J}_{a+}^{n-\alpha} \delta^{n} \mathcal{J}_{a+}^{\beta} \varphi(x) \\
& =\mathcal{J}_{a+}^{n-\alpha} \delta^{n} \mathcal{J}_{a+}^{n-\beta} \mathcal{J}_{a+}^{2 \beta-n} \varphi(x) \\
& =\mathcal{J}_{a+}^{n-\alpha} \mathfrak{D}_{a+}^{\beta} \mathcal{J}_{a+}^{2 \beta-n} \varphi(x) .
\end{aligned}
$$

Then from (2.7.39) of [5] we obtain

$$
{ }^{C} \mathfrak{D}_{a+}^{\alpha} \mathcal{J}_{a+}^{\beta} \varphi(x)=\mathcal{J}_{a+}^{n-\alpha} \mathcal{J}_{a+}^{\beta-n} \varphi(x)=\mathcal{J}_{a+}^{\beta-\alpha} \varphi(x) .
$$

Observe that Theorem 4 is the generalization of Lemma 2.4(i) of [11] where $\beta=\alpha$.

Lemma 9 Let $\alpha \in \mathbb{C}$ with $0<\mathfrak{R}(\alpha) \leq 1$ and $k \in \mathbb{N}$. Let $\varphi(x) \in A C_{\delta}^{n}[a, b], 0<a<b<\infty$. Then

$$
\left(\mathcal{J}_{a+}^{\alpha}\right)^{(k+1)}\left({ }^{C} \mathfrak{D}_{a+}^{\alpha}\right)^{(k+1)} \varphi(x)=\left(\mathcal{J}_{a+}^{\alpha}\right)^{k}\left({ }^{C} \mathfrak{D}_{a^{\alpha}}^{\alpha}\right)^{k} \varphi(x) .
$$

Proof

$$
\begin{aligned}
\left(\mathcal{J}_{a+}^{\alpha}\right)^{(k+1)}\left({ }^{C} \mathfrak{D}_{a+}^{\alpha}\right)^{(k+1)} \varphi(x)= & \left(\mathcal{J}_{a_{+}^{\alpha}}^{\alpha}\right)^{k} \mathcal{J}_{a+}^{\alpha}{ }^{C} \mathfrak{D}_{a_{+}}^{\alpha}\left({ }^{C} \mathfrak{D}_{a+}^{\alpha}\right)^{k} \varphi(x) \\
= & \left(\mathcal{J}_{a^{\alpha}}^{\alpha}\right)^{k}\left(\left({ }^{C} \mathfrak{D}_{a_{+}}^{\alpha}\right)^{k} \varphi(x)-\left({ }^{C} \mathfrak{D}_{a+}^{\alpha}\right)^{k} \varphi(a)\right) \\
& \left(\text { by }(40) \text { with } \Phi(x)=\left({ }^{C} \mathfrak{D}_{a_{+}}^{\alpha}\right)^{k} \varphi(x)\right) \\
= & \left(\mathcal{J}_{a_{+}}^{\alpha}\right)^{k}\left({ }^{C} \mathfrak{D}_{a_{+}}^{\alpha}\right)^{k} \varphi(x) \\
& \left(\text { since }\left({ }^{C} \mathfrak{D}_{a_{+}}^{\alpha}\right)^{k} \varphi(a)=0 \forall k=1,2,3, \ldots\right) .
\end{aligned}
$$


Both Theorem 3 and Lemma 8 deal with the reduction of higher fractional order differential systems to lower order systems for Caputo-Hadamard fractional derivatives. However, in some instances it may also be useful to involve the Caputo-Hadamard and the Hadamard differential operator.

Lemma 10 Let $\varphi(x) \in C_{\delta}^{n}[a, b]$ for some $n \in \mathbb{N}$ and $0<\alpha \leq n$. Then

$$
\mathfrak{D}_{a+}^{n-\alpha C} \mathfrak{D}_{a+}^{\alpha} \varphi(x)=\delta^{n} \varphi(x),
$$

where $\delta=x \frac{d}{d x}$.

Proof

1. If $\alpha \in \mathbb{N}$, then by (19) and from (2.7.13) of [5], (59) becomes

$$
\delta^{n-\alpha} \delta^{\alpha} \varphi(x)=\delta^{n} \varphi(x)
$$

2. Otherwise, since $n=[\Re(\alpha)]+1$, then by definitions

$$
\begin{aligned}
\mathfrak{D}_{a+}^{n-\alpha C} \mathfrak{D}_{a+}^{\alpha} \varphi(x) & =\delta^{[\Re(n-\alpha)]+1} \mathcal{J}_{a+}^{[\mathfrak{R}(n-\alpha)]+1-(n-\alpha)} \mathcal{J}_{a+}^{[\mathfrak{R}(\alpha)]+1-\alpha} \delta^{[\mathfrak{R}(\alpha)]+1} \varphi(x) \\
& =\delta^{n-[\Re(\alpha)]+1} \mathcal{J}_{a+}^{2}\left(\delta^{[\mathfrak{R}(\alpha)]+1} \varphi(x)\right) \\
& =\delta^{n-[\Re(\alpha)]} \delta^{[\mathfrak{R}(\alpha)]} \varphi(x)=\delta^{n} \varphi(x) .
\end{aligned}
$$

\section{Conclusion}

Since the Caputo-Hadamard fractional derivatives were introduced in [11], not much about the modified derivatives were studied despite the fact that the derivatives have many advantages over the usual Hadamard fractional derivative. We proved that the Hadamard fractional derivatives cannot be used to generalize the FTFC whereas the Caputo-Hadamard derivative works perfectly. The FTFC is then used in formulating other results whose applications to fractional vector calculus in the study of Green's theorem, Stoke's theorem and so forth, as well as in the study of anomalous diffusion is a further work. Many new results such as the semigroup properties for the modified derivatives are studied in detail.

\section{Competing interests}

The authors declare that they have no competing interests.

Authors' contributions

All authors contributed equally in this article. They read and approved the final manuscript.

\section{Author details}

${ }^{1}$ Department of Mathematics, Faculty of Sciences, Northwest University, 3099 Kabuga-Katsina Road, Kano, Kano State, Nigeria. ${ }^{2}$ Department of Mathematics and Computer Sciences, Faculty of Arts and Sciences, Cankaya University, Yenimahalle, Ankara, 06810, Turkey. ${ }^{3}$ Department of Logistics Management, Faculty of Management, University of Turkish Aeronautical Association, Etimesgut, Ankara, 06790, Turkey. ${ }^{4}$ Department of Chemical and Materials Engineering, Faculty of Engineering, King Abdulaziz University, Jeddah, Kingdom of Saudi Arabia. ${ }^{5}$ Institute of Space Sciences, P.O. Box MG-23, Magurele, Bucharest 76900, Romania. ${ }^{6}$ Department of Mathematics and Physical Sciences, Prince Sultan University, P.O. Box 66833, Riyadh, 11586, Kingdom of Saudi Arabia.

\section{Acknowledgements}

The first author wishes to give special thanks to His Excellency, the Executive Governor of Kano State of Nigeria, Engineer (Dr.) Rabi'u Musa Kwankwaso, for his endless, patriotic and tireless support and constant encouragement. 


\section{References}

1. Magin, RL: Fractional Calculus in Bioengineering. Begell House, Redding (2006)

2. Baleanu, D, Diethelm, K, Scalas, E, Trujillo, Jj: Fractional Calculus Models and Numerical Methods. Series on Complexity, Nonlinearity and Chaos. World Scientific, Singapore (2012)

3. Hilfer, R (ed.): Applications of Fractional Calculus in Physics. World Scientific, Singapore (2000)

4. Mainardi, F: Fractional Calculus and Waves in Linear Viscoelasticity: An Introduction to Mathematical Models. Imperial College Press, London (2010)

5. Kilbas, AA, Srivastava, HH, Trujillo, JJ: Theory and Applications of Fractional Differential Equations. Elsevier, Amsterdam (2006)

6. Podlubny, I: Fractional Differential Equations. Academic Press, San Diego (1999)

7. Samko, SG, Kilbas, A, Marichev, OI: Fractional Integrals and Derivatives: Theory and Applications. Gordon \& Breach, Amsterdam (1993)

8. Kilbas, AA: Hadamard-type fractional calculus. J. Korean Math. Soc. 38(6), 1191-1204 (2001)

9. Butzer, PL, Kilbas, AA, Trujillo, JJ: Compositions of Hadamard-type fractional integration operators and the semigroup property. J. Math. Anal. Appl. 269, 387-400 (2002)

10. Butzer, PL, Kilbas, AA, Trujillo, JJ: Mellin transform analysis and integration by parts for Hadamard-type fractional integrals. J. Math. Anal. Appl. 270, 1-15 (2002)

11. Jarad, F, Baleanu, D, Abdeljawad, A: Caputo-type modification of the Hadamard fractional derivatives. Adv. Differ. Equ. 2012, $142(2012)$

12. Eliana, CG, De Oliveira, EC: Fractional versions of the fundamental theorem of calculus. Appl. Math. 4, 23-33 (2013)

13. Tarasov, VE: Fractional vector calculus and fractional Maxwell's equations. Ann. Phys. 323, 2756-2778 (2008)

14. Tarasov, VE: Fractional Dynamics: Applications of Fractional Calculus to Dynamics of Particles, Fields and Media. Springer, Heidelberg; Higher Education Press, Beijing (2010)

15. Robert, GB, Sherbert, DR: Introduction to Real Analysis. Wiley, New York (2000)

16. Changpin, LA, Weihua, D: Remarks on fractional derivatives. Appl. Math. Comput. 187, 777-784 (2007)

17. Changpin, LA, Deliang, Q, Yangquan, C: On Riemann-Liouville and Caputo derivatives. Discrete Dyn. Nat. Soc. 2011 Article ID 562494 (2011)

Cite this article as: Gambo et al.: On Caputo modification of the Hadamard fractional derivatives. Advances in Difference Equations 2014, 2014:10

\section{Submit your manuscript to a SpringerOpen ${ }^{\circ}$ journal and benefit from:}

- Convenient online submission

- Rigorous peer review

- Immediate publication on acceptance

- Open access: articles freely available online

- High visibility within the field

- Retaining the copyright to your article 